\title{
SARS-CoV-2 and Hemodialysis: diffusion and mortality in patients and health care team. Reflections from the Lombardy experience
}

\author{
Giuseppe Rombolà ${ }^{1} \cdot$ Marco Heidempergher $^{2} \cdot$ Marina Cornacchiari $^{3} \cdot$ Ivano Baragetti $^{4} \cdot$ Federico Pieruzzi $^{5} \cdot$ The \\ Lombardy-branch of the Italian Society of Nephrology
}

Published online: 1 March 2021

(c) Italian Society of Nephrology 2021

On May 8th, 2020 the Italian Ministry of Health reported that 217,185 people had been infected by SARS-CoV-2 and among them there were 30,201 fatalities. The Case Fatality Rate (CFR), i.e., the ratio between confirmed deaths and confirmed cases, was therefore $13.9 \%$, and the mortality rate (MR), i.e. the ratio between the confirmed deaths and total exposed population, was $0.05 \%$ [1].

Most of these events were recorded in Lombardy, the most densely populated Italian region, where, by April 30th, 2020 , about 460,000 people (4.6\% of the entire regional population) had undergone nasopharyngeal swabs and $17.4 \%$ were positive for COVID-19. Consequently, the CFR was $18.8 \%$, and the MR was $0.15 \%$ [2].

Almost one year later, we know that the clinical spectrum of COVID-19 ranges from a totally asymptomatic infection to mild respiratory tract symptoms to severe pneumonia, multi-organ failure, and death [3]. Preexisting conditions including age $\geq 65$ years, immunodepression, cardiovascular disease, chronic lung disease, diabetes and chronic kidney disease are associated with a worse prognosis [3, 4]. Dialysis patients may present several of these conditions and may be expected to have a dramatically high risk of death in case of COVID-19.

A few days after the appearance of SARS-CoV-2 in Lombardy, 25 dialysis patients from the Bergamo area were found to be affected. Thereafter, all the dialysis centers applied preventive measures, suspected cases were

Giuseppe Rombolà

giuseppe.rombola@asst-settelaghi.it

$1 \quad$ Nephrology ASST Settelaghi, Varese, Italy

2 NephrologyASST-Sacco-Fatebenefratelli, Milano, Italy

3 Nephrology ASST Ovest Milano, Legnano Magenta, Italy

4 NephrologyASST-NordMilano, Cinisello Balsamo, Italy

5 NephrologyASST-Monza-Desio, UniMiB, Monza, Italy isolated, and isolation rooms and pathways were identified for patients who tested positive to nasopharyngeal swabs [5].

By the end of April, the Lombardy branch of the Italian Society of Nephrology launched a survey on SARS-CoV-2 infection in dialysis patients. The survey employed an online questionnaire which was sent to all the dialysis facilities, and information on the effects and diffusion of SARS-CoV-2 infection regarding both patients and health care teams were collected. We report herein the data of this survey, focusing mainly on the mortality of dialysis patients, a population which, regardless of COVID-19, has a mortality rate of about $16 \%$ per year [6].

Our survey reports on 5385 out of 7040 (76.5\%) patients on hemodialysis treatment in the region.

From the beginning of the SARS-CoV-2 outbreak until April 30 th, 2020, a total of 1722 out of 5,385 hemodialysis patients (32.0\%) underwent nasopharyngeal swab, and among those who did, $553(32.1 \%)$ tested positive and 171 of them died (CFR 30.9\%, MR 3.2\%).

Age distribution was similar for patients on dialysis and among the general population (71.6 years on average in Italy) [6]. However, the difference in CFR between dialysis patients and the general population in Lombardy was, 30.9\% compared to $18.8 \%$ (Table 1).

However, there are some factors that weaken the significance of the CFR attributable to COVID-19 in patients on hemodialysis. The probability of finding positive swabs increases with the number of swabs carried out. The number of swabs performed in the general population of Lombardy was much lower than among dialysis patients $(4.6 \%$ vs. $32 \%$, respectively). Most deaths occurred in the first 6 weeks after the outbreak of the disease. Thereafter, although the preventive measures taken in all dialysis centers effectively reduced the spread of SARS CoV-2, in the same period the Italian government imposed a total lockdown, thus drastically reducing the risk of contamination in the general population. 
Table 1 Case Fatality Rate, Mortality Rate and Expected mortality rate in SARS-CoV- 2 negative HDp and general population Lombardy and Italy, in the 60-90-year age group during the SARS-CoV-2 outbreak in Lombardy

\begin{tabular}{lllc}
\hline & $\begin{array}{l}\text { CFR (2 months) SARS-CoV- 2 posi- } \\
\text { tive (\%) }\end{array}$ & $\begin{array}{l}\text { MR (2 months) SARS-CoV- 2 posi- } \\
\text { tive (\%) }\end{array}$ & $\begin{array}{l}\text { Expected MR } \\
\text { SARS-CoV- 2 Nega- } \\
\text { tive (\%) }\end{array}$ \\
\hline HDp & 30.9 & 3.17 & 16.2 \\
Gen. Population Lombardy & 18.8 & 0.15 & 3.32 \\
Gen. Population Italy & 13.6 & 0.045 & 3.45 \\
\hline
\end{tabular}

The annual mortality rate is expressed as \% value; for HDp the values are the historical ones of the Lombardy nephrology register, while for the Lombardy population and the Italian general population the values are those reported by the ISTAT website (http://dati.istat.it/Index.aspx?DataS etCode=DCIS_MORTALITA1) and specifically refer to the 60-90 year age group

In this setting, the MR is probably a more reliable index, and it resulted 21 times higher in the dialysis than in the general population $(3.17 \%$ vs. $0.15 \%$ respectively).

As previously mentioned, dialysis patients have an MR of about $16 \%$ per year [6]. Assuming a homogeneous yearly distribution of mortality rates, 144 out of 5385 dialysis patients would have died within the 2 months of our survey.

In addition to the 171 patients with a positive nasopharyngeal swab who died in the same period, we recorded 60 further deaths in the same period of time, accounting for 231 deaths versus 144 expected deaths.

One of the questions in our survey asked the caregiver nephrologists if they would have been surprised if these patients had died within the following 6 months. Interestingly, $65 \%$ answered that these deaths were likely expected, thus suggesting an acceleration of an event that most likely would have occurred with a relatively short delay.

The same comments highlight how very difficult it is to clearly define what the MR directly related to COVID-19 actually is.

It is interesting to note that many dialysis patients had mild symptoms with an elusive course. This is certainly positive for the affected patient but results in a higher probability of spreading SARS-CoV-2 among patients and caregivers.

As a consequence, we believe that frequent testing is essential to reduce the risk of contamination.

The reasons why dialysis patients, despite the high comorbidity burden, may have less aggressive infection, are not clear; one hypothesis is that a less efficient immune system may increase susceptibility, but the inflammatory reaction may be less intense [7]. In addition, exposure to heparin during dialysis may contribute to mitigating endothelial and pulmonary damage [8].

Our survey also included approximately 1550 nurses and 210 physicians, whose age is much lower than that of dialysis patients. Since the nasopharyngeal swab was mostly done in symptomatic cases, asymptomatic infections presumably escaped. Within this limit, 34 out of 210 physicians and 90 out of 1,550 nurses tested positive $(7.0 \%$ of all health care personnel). Dialysis centers are overcrowded places where patients, nurses, transport workers, cleaning staff, and doctors work together for many hours a week. In 33 of the 37 dialysis centers that took the survey the dialysis nurses affected by COVID-19 ranged from none to 2 , while in 4 centers there were up to 20 symptomatic nurses confined at home, thereby compromising the dialysis organization.

However, by May 22nd, 81,261 health workers had been tested for the presence of specific IgG antibodies against SARS-CoV-2 (Liaison SARS-CoV-2 IgG; DiaSorin, Italy) in Lombardy and 11,295 (13.9\%) of them were found positive [9].

This means that we could assume that at least 121 additional dialysis healthcare workers could have been infected and asymptomatic without being diagnosed or isolated.

Three main lessons may be learned from this experience.

1. It would be useful to include versatile rooms in the construction project of any new dialysis facility: for example small 3-bed rooms with dedicated access that could be quickly and easily converted into quarantine chambers to dialyze infected patients during pandemics.

2. Future health crisis planning should foresee an adequate number of doctors and nurses trained to handle life-saving therapies such as dialysis.

3. Regularly test dialysis patients and healthcare professionals, even if they are asymptomatic, to prevent viral spread and to guarantee the efficiency of the facility.

\section{Appendix}

List of dialysis centers and contact persons for each center that participated to the survey, shared data and discussed the conclusions.

P. Fabbrini, ASST Nord Milano; S. Badalamenti H. Umanitas-Rozzano, Mi; S. Bertoli Multimedica Milano; S. Bisegna, ASST Melegnano-Martesana, M. Bracchi, ASST Franciacorta; A. Castiglioni, ASST ValleOlona; C. Colturi, ASST Valtellina; E. Costantino, ASST Garda; M. Cozzolino, 
ASST Santi Paolo e Carlo; G. De Petri, ASST Crema; M. Doria, Policlinico San Donato; C. Esposito, H. Fondazione Maugeri, Pavia; M. Farina, ASST Lodi; E. Galli, ASST Bergamo Ovest-Treviglio; M. Gallieni, ASST Sacco-Fatebenefratelli Mi; A. Gazo, ASST-Pavia-Vigevano; C. Guastoni, ASST Ovest Milano; F. Ledda, H Umanitas-Gavazzeni, Bergamo;M. Lorenz, Policlinico San Marco, Zingonia; G. Mazzola, ASST Mantova; G. Melfa, ASST-Como; P. Messa, ASST-Policlinico Milano; A. Materangelis, Policlinico Ponte San Pietro, Bergamo; F. Milanesi, ASST Pavia Voghera; E. Minetti ASST Grande ospedale metropolitano, Milano; G.Montini, Fondazione Ca Granda Policlinico, Milano; P.Ondei, ASST Papa Giovanni XXIII, Bergamo; F. Pieruzzi, ASST Monza e Desio; T. Rampino Fondazione Policlinico San Matteo, Pavia; R. Floreani ASST-Settelaghi, Varese; R. Savino, ASST Rhodense; P. Scalia, ASST Settelaghi, Tradate; G. Slaviero, H. San Raffaele, Milano.

Acknowledgments The Authors thank all the colleagues who participated in this survey and express their recognition particularly towards those who were in the line of fire of the SARS-CoV-2 pandemic and sadly witnessed the death of their patients.

The Lombardy-branch of the Italian Society of Nephrology: Giuseppe Rombolà, Marco Heidempergher, Marina Cornacchiari, Ivano Baragetti, Federico Pieruzzi.

\section{References}

1. Covid-19 - Situazione in Italia. http://www.salute.gov.it/portale/ nuovocoronavirus/dettaglioContenutiNuovoCoronavirus.jsp?lingu

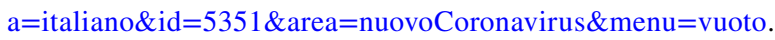
Accessed 10 Sept 2020

2. Coronavirus in Italia, i dati e la mappa. https://lab24.ilsole24or e.com/coronavirus/. Accessed 20 June 2020

3. Wang D et al (2020) Clinical characteristics of 138 hospitalized patients with 2019 novel coronavirus-infected pneumonia in Wuhan, China. JAMA J Am Med Assoc 323:1061-1069

4. Information for Healthcare Professionals about Coronavirus (COVID-19). CDC. https://www.cdc.gov/coronavirus/2019-ncov/ hcp/index.html. Accessed 20 June 2020

5. Rombolà $\mathrm{G}$ et al (2020) Practical indications for the prevention and management of SARS-CoV-2 in ambulatory dialysis patients: lessons from the first phase of the epidemics in Lombardy. $\mathrm{J}$ of Neph 33:193-196

6. Registro Italiano di Dialisi e Trapianto (Lombardia 2018). www. sin-ridt.org. Accessed 20 June 2020

7. Ma Y et al (2020) Epidemiological, clinical, and immunological features of a cluster of COVID-19 contracted hemodialysis patients. Kidney Int Reports 5(8):1333-1341

8. Paranjpe I et al (2020) Association of treatment dose anticoagulation with in-hospital survival among hospitalized patients with COVID-19. J Am Coll Cardiol. 76(1):122-124

9. 124.000 test sierologici su sanitari e cittadini in quarantena. 22 May 2020. https://www.lombardianotizie.online/covid-test-siero logici/. Accessed 20 June 2020

Publisher's Note Springer Nature remains neutral with regard to jurisdictional claims in published maps and institutional affiliations. 